K.-I. Yoshida

Nagoya Math. J.

Vol. 147 (1997), 179-191

\title{
COFINITENESS OF LOCAL COHOMOLOGY MODULES FOR IDEALS OF DIMENSION ONE
}

\author{
KEN-ICHI YOSHIDA
}

\begin{abstract}
In this paper, we prove that for any ideal $I$ of dimension one $\mathrm{H}_{I}^{2}(M)$ is $I$-cofinite for all $i$ and for any finite $A$-module $M$. Furthermore, for any ideal $I$ over any regular local ring $A$, we investigate the relationship between $I$-cofiniteness and vanishing for local cohomology modules $\mathrm{H}_{I}^{2}(M)$.
\end{abstract}

\section{§1. Main Theorem}

Let $A$ be a Noetherian ring. For an ideal $I$ of $A$ and an $A$-module $M$, we set

$$
\mathrm{H}_{I}^{\imath}(M)=\lim _{k} \operatorname{Ext}_{A}^{i}\left(A / I^{k}, M\right) .
$$

In general, a functor $\mathrm{H}_{I}^{\imath}(-)$ is the $i^{\text {th }}$ right derived functor of the functor

$$
\Gamma_{I}(-)=\lim _{k} \operatorname{Hom}_{A}\left(A / I^{k},-\right) .
$$

Let $A$ be a Noetherian local ring, with maximal ideal $\mathfrak{m}$, and residue field $k=A / \mathfrak{m}$. If $M$ is a finite $A$-module, then $\operatorname{Supp}_{A}\left(\mathrm{H}_{\mathfrak{m}}^{j}(M)\right) \subseteq V(\mathfrak{m})$ and $\operatorname{Ext}_{A}^{2}\left(A / \mathfrak{m}, \mathrm{H}_{\mathfrak{m}}^{\jmath}(M)\right)$ is of finite type for all $i, j$; see e.g. Huneke and Koh [11, Remark 1.3, 2.1].

Grothendieck [6] made the following conjecture:

If $I$ is an ideal of $A$ and $M$ is a finite $A$-module, then $\operatorname{Hom}_{A}\left(A / I, \mathrm{H}_{I}^{i}(M)\right)$ is of finite type for all $i$.

This conjecture is false in general. In fact, Hartshorne [8] gave the following counterexample.

Let $k$ be a field and let $A=k[[X, Y, Z, W]], I=(X, Z) A$. We put $M=A /(X Y-Z W) A$. Then $\operatorname{Hom}_{A}\left(A / I, \mathrm{H}_{I}^{2}(M)\right)$ is not of finite type.

Hartshorne [8] defined an $A$-module $W$ to be $I$-cofinite if $\operatorname{Supp}_{A}(W) \subseteq$ $V(I)$ and $\operatorname{Ext}_{A}{ }^{2}(A / I, W)$ is of finite type for all $i$.

Received April 28, 1995. 
Noting that $\operatorname{dim} A / I=2$ in the above example, we ask the following question:

Let $A$ be a local ring and $I$ an ideal of $A$ such that $\operatorname{dim}_{A} A / I=1$. Let $M$ be a finite A-module. Then when is $\mathrm{H}_{I}^{2}(M) I$-cofinite for all $i$ ?

With respect to this question, Hartshorne [8, Corollary 7.7] showed if $A$ is a complete regular local ring and $I$ is a prime ideal such that $\operatorname{dim}_{A} A / I=$ 1 then $\mathrm{H}_{I}^{i}(M)$ is $I$-cofinite for any finite $A$-module $M$. Huneke and Koh [11, Theorem 4.1] proved that if $A$ is a complete Gorenstein local domain and $I$ is an ideal of $A$ such that $\operatorname{dim}_{A} A / I=1$ then $\operatorname{Ext}_{A}^{i}\left(N, \mathrm{H}_{I}^{j}(M)\right)$ is of finite type for any finite $A$-modules $M, N$ such that $\operatorname{Supp}_{A}(N) \subseteq V(I)$ and for all $i, j$. Furthermore, using [11, Theorem 4.1], Delfino [3] proved that if $A$ is a complete local domain under some mild conditions then the similar result holds.

In this note, refining their proof, we prove the following theorem.

TheOrem 1.1. Let $A$ be a local ring and $I$ an ideal with $\operatorname{dim} A / I=1$. Let $M$ be a finite $A$-module. Then for any finite $A$-module $N$ such that $\operatorname{Supp}_{A}(N) \subseteq V(I)$, we have $\operatorname{Ext}_{A}^{i}\left(N, \mathrm{H}_{I}^{j}(M)\right)$ is of finite type for all $i, j$.

In order to prove Theorem 1.1, we need fundamental two lemmas.

Lemma 1.2. (Delfino [3, Lemma 2], Huneke and Koh [11, Lemma 4.2]) Let $A$ be a Noetherian ring and $I$ an ideal of $A$. Let $p \geq 0$ be an integer. Then for an $A$-module $V$, the following conditions are equivalent.

(1) $\operatorname{Ext}_{A}^{t}(A / I, V)$ is a finite $A$-module for all $t \leq p$.

(2) $\operatorname{Ext}_{A}^{t}(A / \sqrt{I}, V)$ is a finite A-module for all $t \leq p$.

(3) $\operatorname{Ext}_{A}^{t}(N, V)$ is a finite A-module for any finite $A$-module $N$ such that $\operatorname{Supp}_{A}(N) \subseteq V(I)$ and for all $t \leq p$.

The following lemma has been essentially proved by Hartshorne [8].

LEMma 1.3. (cf. Hartshorne [8]) Let $R$ be a complete regular local ring and $J$ a radical ideal of $R$ such that $\operatorname{dim} R / J=1$. Let $M$ be a finite $R$-module. Then $\operatorname{Ext}_{R}^{i}\left(R / J, \mathrm{H}_{J}^{j}(M)\right)$ is of finite type for all $i, j$.

Huneke and Koh [11] proved a stronger result than the above lemma using the Local Lichtenbaum-Hartshorne Theorem (cf. [2]). Furthermore, 
Delfino [3] proved the similar result as Proposition 1.4 (see below) and he has reduced the proof of the case of complete local domains to that of complete Gorenstein local domains using noetherian normalization.

Our original idea is to reduce directly the proof of general case to that of complete regular local rings using Cohen's structure theorem. Our main tool is the following proposition.

Proposition 1.4. Let $R$ be a local ring and let $J, \mathfrak{a}$ be ideals of $R$. We set $A=R / \mathfrak{a}$ and $I=J A$. Then for any $A$-module $W$, the following conditions are equivalent:

(1) $\operatorname{Ext}_{R}^{i}(R / J, W)$ is a finite $R$-module for all $i$.

(2) $\operatorname{Ext}_{A}^{i}(A / I, W)$ is a finite $A$-module for all $i$.

Proof. We consider the following spectral sequence

$$
\left.E_{2}^{p q}=\underset{A}{\operatorname{Ext}} \stackrel{R}{\operatorname{Tor}}(A, R / J), W\right) \Longrightarrow E^{n}=\operatorname{Ext}_{R}^{n}(R / J, W)
$$

see [17, Theorem 11.65].

$(1) \Longrightarrow(2)$ : (cf. [3, Theorem 3]) By the assumption, $E^{n}=\operatorname{Ext}_{R}^{n}(R / J$, $W$ ) is of finite type for each $n$. In order to see (2), it suffices to show that $E_{2}^{p q}$ is of finite type for all $p, q$. We prove this by induction on $p$.

First, suppose $p=0$. Note that $E_{2}^{00} \cong \operatorname{Hom}_{R}(A / I, W)$ is of finite type by Lemma 1.2. Moreover, since $\operatorname{Supp}_{A}\left(\operatorname{Tor}_{q}^{R}(A, R / J)\right) \subseteq V(I), E_{2}^{0 q}=$ $\operatorname{Hom}_{A}\left(\operatorname{Tor}_{q}^{R}(A, R / J), W\right)$ is of finite type for all $q$ (cf. Lemma 1.2).

Next, suppose $p=1$. Since $E_{2}^{10} \cong E_{\infty}^{10}$ is a submodule of $E^{1}$, it is also of finite type. By the above argument, we obtain that $E_{2}^{1 q}$ is of finite type for all $q$.

Finally, suppose that for an integer $t \geq 2, E_{2}^{p q}$ is a finite $A$-module for all $0 \leq p<t$ and for all $q$. Then we must show that $E_{2}^{t q}$ is of finite type for all $q$. By Lemma 1.2, it is enough to show that $E_{2}^{t 0}$ is of finite type.

For each $p<t$, since $E_{r}^{p q}$ is a subquotient of $E_{2}^{p q}$ for all $r \geq 3$, it is of finite type. On the other hand, since we have

$$
E_{r}^{t 0}=E_{r-1}^{t 0} / \operatorname{Im} d_{r-1}^{t-r+1, r-2}
$$

and

$$
\operatorname{Im} d_{r-1}^{t-r+1, r-2} \cong E_{r-1}^{t-r+1, r-2} / \operatorname{ker} d_{r-1}^{t-r+1, r-2}
$$


we get that $E_{r}^{t 0}$ is of finite type if and only if so is $E_{r-1}^{t 0}$. Furthermore, as $E_{\infty}^{t 0}$ is a submodule of $E^{t}$, it is of finite type. Therefore we conclude that $E_{2}^{t 0}$ is of finite type as required.

$(2) \Longrightarrow(1)$ : By the assumption, $E_{2}^{p 0}$ is of finite type for all $p$. Thus by Lemma 1.2, we get $E_{2}^{p q}$ is also of finite type for all $p, q$, and so is $E_{r}^{p q}$ for all $r \geq 3$, because $E_{r}^{p q}$ is a subquotient of $E_{2}^{p q}$. Therefore $E_{\infty}^{p q}$ is of finite type.

On the other hand, for each $n$, since $E^{n}=\operatorname{Ext}_{R}^{n}(R / J, W)$ has the finite filtration as follows:

$$
E^{n}=E_{0}^{n} \supseteq E_{1}^{n} \supseteq \cdots \supseteq E_{n}^{n} \supseteq E_{n+1}^{n}=0, \quad E_{p}^{n} / E_{p+1}^{n} \cong E_{\infty}^{p, n-p} .
$$

Hence we conclude that $E^{n}$ is of finite type for all $n$.

Using this proposition, we now prove Theorem 1.1. By Lemma 1.2, we may assume that $I=\sqrt{I}$. Let $M$ be a given finite $A$-module.

First, suppose that $A$ is complete. Then we can write as $A=R / \mathfrak{a}$, where $R$ is a complete regular local ring and $\mathfrak{a}$ an ideal of $R$ by Cohen's structure theorem. Moreover, when we write as $I=J / \mathfrak{a}$, we get $\operatorname{dim} R / J=$ $\operatorname{dim} A / I=1$. Thus from Lemma 1.2, $\operatorname{Ext}_{R}^{i}\left(R / J, \mathrm{H}_{J}^{j}(M)\right)$ is a finite $R$ module for all $i, j$. Hence the required assertion follows from Proposition 1.4 and Lemma 1.3.

In general case, stated as above, $\operatorname{Ext}_{A}^{\imath}\left(A / I, \mathrm{H}_{I}^{j}(M)\right) \otimes \widehat{A}$ is of finite type for all $i, j$, where $\widehat{A}$ be an $\mathrm{m}$-adic completion of $A$. Since $\widehat{A}$ is faithfully flat over a noetherian local ring $A$, the required assertion follows from this.

Remark 1. The proof of Proposition 1.4 is inspired by the proof of Delfino [3, Theorem 3].

We shall give one more application of Proposition 1.4. Before stating our result, we recall the definition of ara $(I)$, the arimetical rank of $I$.

$$
\operatorname{ara}(I)=\inf \left\{n \mid \exists a_{1}, a_{2}, \ldots, a_{n} \in A \text { such that } \sqrt{\left(a_{1}, \ldots, a_{n}\right)} A=\sqrt{I}\right\} .
$$

In case where $\operatorname{ara}(I) \leq 1$, we obtain the following result.

Corollary 1.5. (cf. Hartshorne [8, Corollary 6.3]) Let $A$ be a local ring and $I$ an ideal of $A$ with $\operatorname{ara}(I) \leq 1$. Let $M$ be any finite A-module. Then for any finite $A$-module $N$ such that $\operatorname{Supp}_{A}(N) \subseteq V(I)$, we have $\operatorname{Ext}_{A}^{i}\left(N, \mathrm{H}_{I}^{j}(M)\right)$ is of finite type for all $i, j$. 
Proof. By Lemma 1.2, we may assume that $I=a A$. Moreover, we may assume that $A$ is complete. Then we can write as $A=R / \mathfrak{a}$ and $I=r A$, where $R$ is a complete regular local ring and $r \in R$. Replacing $A$ and $I=a A$ with $R$ and $J=r R$, we may assume that $A$ is a complete regular local ring (cf. Proposition 1.4). Then the required assertion follows from Hartshorne [8, Corollary 6.3].

In general, when $\operatorname{dim} A / I=2$, we have the counterexample (for cofiniteness) given by Hartshorne. So we consider the following question.

QUESTION 1.6. Let $A$ be a regular local ring and $I$ an ideal of $A$. Then when is $\mathrm{H}_{I}^{i}(A)$ I-cofinite for all $i$ ?

For this question, we can give the following answer.

TheOREM 1.7. Let $A$ be a regular local ring and $I$ an ideal of $A$ with $h=$ height $I$. Then the following conditions are equivalent:

(1) $\operatorname{Hom}_{A}\left(A / I, \mathrm{H}_{I}^{i}(A)\right)$ is of finite type for all $i \geq h+1$.

(2) $\mathrm{H}_{I}^{i}(A)=0$ for all $i \neq h$.

When this is the case, $A / I$ is equidimensional and $\mathrm{H}_{I}^{h}(A)$ is I-cofinite.

In section 2, we give a proof of this theorem. Further, in section 3, for any local ring, we investigate an equivalence among the following three conditions and give a necessary condition for which the last two conditions are equivalent.

(1) $\operatorname{Hom}_{A}\left(A / I, \mathrm{H}_{I}^{\imath}(A)\right)$ is of finite type for all $i \geq h+1$, where $h=$ height $I$.

(2) $\mathrm{H}_{I}^{i}(A)$ is of finite type for all $i \geq h+1$.

(3) $\mathrm{H}_{I}^{i}(A)=0$ for all $i \geq h+1$.

\section{$\S 2$. Vanishing and cofiniteness of local cohomology}

In this section, we prove Theorem 1.7. Before proving this, we recall the following two theorems.

TheOREm 2.1. (Call and Sharp [2]) Let $A$ be a local ring of dimension $d$ and $I$ an ideal of $A$. If $\operatorname{dim} \widehat{A} / I \widehat{A}+P \geq 1$ for all minimal prime ideal $P$ of $\widehat{A}$ of dimension $d$, then $\mathrm{H}_{I}^{d}(A)=0$. 
The above theorem is called The Local Lichtenbaum-Hartshorne Theorem.

Theorem 2.2. (Huneke and Koh [11, Theorem 2.3]) Let $A$ be a regular local ring and $I$ an ideal of $A$. Let $r>\operatorname{bight}(I)$ be a given integer, where

$$
\text { bight } I=\max \left\{\text { height } P \mid P \in \operatorname{Min}_{A}(A / I)\right\} \text {. }
$$

If $\operatorname{Hom}_{A}\left(A / I, \mathrm{H}_{I}^{i}(A)\right)$ is of finite type for all $i \geq r$, then $\mathrm{H}_{I}^{i}(A)=0$ for all $i \geq r$.

Using these theorems, we prove Theorem 1.7.

Proof of Theorem 1.7. We may assume that $I=\sqrt{I}$ by Lemma 1.2. We set $b(\mathfrak{a})=$ bight $\mathfrak{a}-$ height $\mathfrak{a}$ for any ideal $\mathfrak{a}$ of $A$.

Suppose (1). First we prove that $A / I$ is equidimensional, that is, $b(I)=$ 0 . We assume that $b=b(I)>0$, and the result holds for any ideal a such that $b(\mathfrak{a})<b$. We can write as $I=U \cap J$, where

$$
U=\bigcap\left\{P \mid P \in \operatorname{Min}_{A}(A / I), \text { height } P<\operatorname{bight} I\right\} \subseteq \mathfrak{m}
$$

and

$$
J=\bigcap\left\{P \mid P \in \operatorname{Min}_{A}(A / I), \text { height } P=\operatorname{bight} I\right\} \subseteq \mathfrak{m} .
$$

Localizing at a prime ideal $P \in \operatorname{Min}_{A}(A / U+J)$, we may assume $\sqrt{U+J}=$ m. Note that $1 \leq \operatorname{dim} A / J<\operatorname{dim} A / U$.

From Theorem 2.1 and the Mayer-Vietoris sequence for local cohomology, we get

$$
\begin{aligned}
0=\mathrm{H}_{\mathfrak{m}}^{d-1}(A) \longrightarrow \mathrm{H}_{U}^{d-1}(A) \oplus & \mathrm{H}_{J}^{d-1}(A) \\
& \longrightarrow \mathrm{H}_{I}^{d-1}(A) \longrightarrow \mathrm{H}_{\mathfrak{m}}^{d}(A) \cong E_{A} \longrightarrow 0 \quad(\mathrm{ex}) .
\end{aligned}
$$

Since $\operatorname{Hom}_{A}\left(A / U, \mathrm{H}_{U}^{d-1}(A)\right) \subseteq \operatorname{Hom}_{A}\left(A / I, \mathrm{H}_{I}^{d-1}(A)\right) \quad$ is of finite type and bight $U<d-1$, we have $\mathrm{H}_{U}^{d-1}(A)=0$ by Theorem 2.2 .

Now suppose $\operatorname{dim} A / J \geq 2$. Then bight $J=$ height $J \leq d-2$. By the above argument, we obtain that $\mathrm{H}_{J}^{d-1}(A)=0$, and so that $\mathrm{H}_{I}^{d-1}(A) \cong$ $E_{A}$. However, since $\widehat{A} / I \widehat{A} \cong \operatorname{Hom}_{\widehat{A}}\left(\operatorname{Hom}_{\widehat{A}}\left(\widehat{A} / I \widehat{A}, E_{\widehat{A}}\right), E_{\widehat{A}}\right)$ is not of finite length, we get a contradiction. Thus we have $\operatorname{dim} A / J=1$. 
Applying the functor $\operatorname{Hom}_{A}(A / J,-)$ to the above exact sequence, we get

$$
\begin{aligned}
& 0 \rightarrow \operatorname{Hom}_{A}\left(A / J, \mathrm{H}_{J}^{d-1}(A)\right) \rightarrow \operatorname{Hom}_{A}\left(A / J, \mathrm{H}_{I}^{d-1}(A)\right) \\
& \rightarrow \operatorname{Hom}_{A}\left(A / J, E_{A}\right) \rightarrow \operatorname{Ext}_{A}^{1}\left(A / J, \mathrm{H}_{J}^{d-1}(A)\right) \\
& \rightarrow \operatorname{Ext}_{A}^{1}\left(A / J, \mathrm{H}_{I}^{d-1}(A)\right) \rightarrow 0 \quad(\mathrm{ex}) \text {. }
\end{aligned}
$$

From Lemma 1.3, we have that $\operatorname{Ext}_{A}^{2}\left(A / J, \mathrm{H}_{J}^{d-1}(A)\right)$ is of finite type for all $i$. Moreover, the assumption implies that $\operatorname{Hom}_{A}\left(A / J, \mathrm{H}_{I}^{d-1}(A)\right)$ is of finite type, and so is $\operatorname{Hom}_{A}\left(A / J, E_{A}\right)$. This is a contradiction. Therefore we conclude that $I$ is unmixed, that is, bight $I=$ height $I$.

Hence by Theorem 2.2, we have $\mathrm{H}_{I}^{i}(A)=0$ for all $i>h$. On the other hand, as grade $I=h$, we have $\mathrm{H}_{I}^{i}(A)=0$ for all $i<h$, and we thus get the statement (2).

Conversely, suppose (2). Then the statement (1) trivially follows from this. Furthermore, we now consider a spectral sequence

$$
E_{2}^{p q}=\underset{A}{\operatorname{Ext}}\left(A / I, \mathrm{H}_{I}^{q}(A)\right) \Longrightarrow E^{n}=\operatorname{Ext}_{A}^{n}(A / I, A) .
$$

From the assumption (2), this spectral sequence collapses and we have $E_{2}^{p h} \cong E^{p+h}$. In particular, $E_{2}^{p h}=\operatorname{Ext}_{A}^{p}\left(A / I, \mathrm{H}_{I}^{h}(A)\right)$ is of finite type for all $p$.

The next result follows from this.

COROLlary 2.4. Under the same notations as in Theorem 1.7, if $\operatorname{Hom}_{A}\left(A / I, \mathrm{H}_{I}^{i}(A)\right)$ is of finite type for all $i \geq h+1$, then $\operatorname{Spec}(A / I)$ is connected in codimension one.

Proof. It is enough to show that for any prime ideal $P \in V(I)$ such that height $(P / I)>1, \operatorname{Spec}(A / I)_{P} \backslash\left\{P A_{P}\right\}$ is connected (cf. Hartshorne [7, Proposition 1.3]). In this proof, we may assume that $P=\mathfrak{m}$ and $\operatorname{dim} A / I \geq$ 2. Then by the assumption, we have $\mathrm{H}_{I}^{d-1}(A)=\mathrm{H}_{I}^{d}(A)=0$. Since $A$ is regular, we thus get that $\operatorname{Spec}(A / I) \backslash\{\mathfrak{m}\}$ is connected as required; see the proof of Huneke and Lyubeznik [12, Theorem 2.9].

Remark 2. Hochster and Huneke [9] proved the following theorem which is a generalization of Faltings' connectedness theorem. 
THEOREM 2.5. (cf. [9, Theorem 3.3]) Let $A$ be a complete equidimensional local ring. Suppose that $\mathrm{H}_{\mathfrak{m}}^{d}(A)$ is indecomposable. Then for any ideal $I$ such that $\mathrm{H}_{I}^{d-1}(A)=\mathrm{H}_{I}^{d}(A)=0$, the punctured spectrum of $A / I$ is connected.

When $\operatorname{dim} A / I=2$, from Huneke and Lyubeznik [12, Theorem 2.9], we obtain the following result.

Corollary 2.6. (cf. Huneke and Koh [11, Theorem 3.6]) Let $A=$ $k\left[\left[x_{1}, \ldots, x_{d}\right]\right]$, where $k$ is a separably closed field and $d \geq 2$. Then for any ideal $I$ of $A$ such that $\operatorname{dim} A / I=2$, the following conditions are equivalent:

(1) $\operatorname{Hom}_{A}\left(A / I, \mathrm{H}_{I}^{d-1}(A)\right)$ is of finite type.

(2) $\mathrm{H}_{I}^{d-1}(A)=0$.

(3) $\operatorname{Spec}(A / I) \backslash\{\mathfrak{m}\}$ is connected.

When this is the case, $\mathrm{H}_{I}^{i}(A)=0$ for all $i \neq d-2$ and $\mathrm{H}_{I}^{d-2}(A)$ is I-cofinite.

When height $I=1$, Theorem 1.7 implies that the following result.

Corollary 2.7. Let $A$ be a regular local ring and $I$ an ideal of $A$ with height $I=1$. Then the following conditions are equivalent:

(1) $\operatorname{Hom}_{A}\left(A / I, \mathrm{H}_{I}^{i}(A)\right)$ is of finite type for all $i \geq 2$.

(2) $A / I$ is equidimensional.

(3) $\operatorname{ara}(I)=1$.

(4) $\mathrm{H}_{I}^{i}(A)=0$ for all $i \neq 1$.

Remark 3. Let $A$ be a local ring and $I$ an ideal of $A$ with $h=$ height $I$. Then the following statements hold.

(1) If $I$ is a set-theoretic complete intersection, that is, $\operatorname{ara}(I)=h$, then $\mathrm{H}_{I}^{i}(A)=0$ for all $i \neq h$.

(2) (cf. Peskine and Szpiro [16, Chapitre III, Proposition 4.1]) If $A$ is a regular local ring of $\operatorname{char}(A)=p>0$ and $A / I$ is Cohen-Macaulay, then $\mathrm{H}_{I}^{i}(A)=0$ for all $i \neq h$. 
(3) (cf. Huneke and Koh [11]) Let $A=k\left[\left[x_{1}, \ldots, x_{6}\right]\right]$, where $k$ is a field of $\operatorname{char}(k)=0$ and set

$$
P=I_{2}\left(\begin{array}{lll}
x_{1} & x_{2} & x_{3} \\
x_{4} & x_{5} & x_{6}
\end{array}\right) .
$$

Then $P$ is a Cohen-Macaulay prime ideal with height $P=2, \operatorname{dim} A / P$ $=4$ and $\mathrm{H}_{P}^{3}(A) \neq 0$.

\section{$\S 3$. Vanishing and finiteness of local cohomology}

In the previous section, we proved the following fact (cf. Theorem 1.7).

If $A$ is a regular local ring and $I$ an ideal of $A$ with $h=$ height $I$, then the following conditions are equivalent.

(1) $\operatorname{Hom}_{A}\left(A / I, \mathrm{H}_{I}^{i}(A)\right)$ is of finite type for all $i \geq h+1$.

(2) $\mathrm{H}_{I}^{i}(A)$ is of finite type for all $i \geq h+1$.

(3) $\mathrm{H}_{I}^{i}(A)=0$ for all $i \geq h+1$.

When this is the case, we have $A / I$ is equidimensional, $\operatorname{Spec}(A / I)$ is connected in codimension one and $\mathrm{H}_{I}^{h}(A)$ is $I$-cofinite.

For any local ring $A,(3) \Longrightarrow(2) \Longrightarrow(1)$ is true, but $(2) \Longrightarrow(1)$ is not always true; see Example 3.2 below. On the other hand, $(2) \Longrightarrow(3)$ is always true. In fact, we can prove the following proposition.

Proposition 3.1. Let $A$ be a local ring and $I$ an ideal of $A$. Let $M$ be a finite $A$-module. If $\mathrm{H}_{I}^{i}(M)$ is of finite type for all $i \geq r$ for some $r \geq 1$, then $\mathrm{H}_{I}^{i}(M)=0$ for all $i \geq r$.

Proof. We prove by induction on $d=\operatorname{dim} M$. If $d=0$, the assertion is clear. Suppose $d>0$ and $\mathrm{H}_{I}^{i}(M)=0$ for all $i>r$. It is enough to show $\mathrm{H}_{I}^{r}(M)=0$.

First suppose depth $M>0$. Take $x \in \mathfrak{m}$ which is $M$-regular and set $\bar{A}=A / x A, \bar{I}=I \bar{A}$ and $\bar{M}=M / x M$. Then we can get as follows:

$$
\begin{aligned}
\mathrm{H}_{I}^{r}(M) \stackrel{x}{\rightarrow} \mathrm{H}_{I}^{r}(M) \rightarrow & \mathrm{H}_{\bar{I}}^{r}(\bar{M}) \rightarrow \mathrm{H}_{I}^{r+1}(M)=0 \quad(\mathrm{ex}) \\
\mathrm{H} \frac{i}{I}(\bar{M}) & =0 \quad \text { for all } i>r .
\end{aligned}
$$


Hence by induction hypothesis, we get $\mathrm{H}_{\bar{I}}^{r}(\bar{M})=0$. Thus we have $\mathrm{H}_{I}^{r}(M)=0$ by Nakayama's lemma.

Next suppose depth $M=0$. Put $W=\mathrm{H}_{\mathfrak{m}}^{0}(M)$. Since $W$ is of finite length, we have $\mathrm{H}_{I}^{0}(W)=W$ and $\mathrm{H}_{I}^{i}(W)=0$ for all $i \geq 1$. Hence we get $\mathrm{H}_{I}^{i}(M) \cong \mathrm{H}_{I}^{i}(M / W)$ for all $i \geq 1$, and so we get the required assertion from the above argument.

ExAmple 3.2. Let $A=k\left[\left[x_{1}, \ldots, x_{n}\right]\right] /\left(x_{1} x_{2} \cdots x_{n}\right), I=\left(x_{2}, \ldots, x_{n}\right) A$ and $P=x_{1} A$, where $k$ is a field and $n \geq 2$ is an integer. Put $d=n-1=$ $\operatorname{dim} A$. Then $A$ is a complete Gorenstein local $\operatorname{ring}$ and $\operatorname{dim} A / I=1$ and height $I=d-1$. In particular, $\operatorname{Hom}_{A}\left(A / I, \mathrm{H}_{I}^{d}(A)\right)$ is a finite $A$-module by Theorem 1.1. However, we get $\mathrm{H}_{I}^{d}(A) \neq 0$ by 'The Local LichtenbaumHartshorne Theorem,' because $\operatorname{dim} A / I+P=0$.

The next result corresponds to Theorem 1.7.

Proposition 3.3. Suppose that $A$ is (F.L.C.), that is, $\mathrm{H}_{\mathfrak{m}}^{i}(A)$ is a finite $A$-module for all $i<\operatorname{dim} A$. Then the following conditions are equivalent.

(1) $\mathrm{H}_{I}^{2}(A)$ is of finite type for all $i \geq h+1$.

(2) $\mathrm{H}_{I}^{i}(A)=0$ for all $i \geq h+1$.

When this is the case, $A / I$ is equidimensional. If, in addition, $A$ is CohenMacaulay, then $\mathrm{H}_{I}^{h}(A)$ is $I$-cofinite and $\operatorname{Spec}(A / I)$ is connected in codimension one.

In order to prove this, we need the following theorem which was proved by Faltings.

Theorem 3.4. (Faltings [4], [10, Theorem 3.2]) Let $A$ be a local ring which admits a dualizing complex. For any ideal $I$ of $A$ and for any finite A-module $M$, we set

$$
s(I, M)=\min \left\{\operatorname{depth} M_{P}+\operatorname{height}\left(\frac{I+P}{P}\right) \mid P \in \operatorname{Spec}(A) \backslash V(I)\right\} .
$$

If $j<s(I, M)$, then $\mathrm{H}_{I}^{j}(M)$ is of finite type. If $j=s(I, M)$, then $\mathrm{H}_{I}^{j}(M)$ is not of finite type. 
Remark 4. If $A$ is (F.L.C.), then $A_{P}$ is a Cohen-Macaulay local ring and

$\operatorname{dim} A=\operatorname{dim} A_{P}+\operatorname{dim} A / P \quad$ for every $P \in \operatorname{Spec} A \backslash\{\mathfrak{m}\}$.

Corollary 3.5. Suppose that $A$ is (F.L.C.) and $h=$ height $I \geq 1$. Then $h=s(I, A)$ and $\mathrm{H}_{I}^{h}(A)$ is not of finite type.

Proof. For any prime ideal $P \in \operatorname{Spec}(A) \backslash V(I)$, we get

$$
\begin{aligned}
& \operatorname{depth} A_{P}+\text { height }\left(\frac{I+P}{P}\right) \\
& \quad=\operatorname{dim} A_{P}+\operatorname{dim} A / P-\operatorname{dim}(A / I+P) \quad(\text { cf. }[14, \text { Theorem 31.6]) } \\
& \quad=\operatorname{dim} A-\operatorname{dim}(A / I+P) \\
& \quad=\operatorname{height}(I+P) \geq h .
\end{aligned}
$$

Hence we have $s(I, A) \geq h$.

We now show that the inverse inequality holds. Take a prime ideal $P \in \operatorname{Assh}_{A}(A / I)$ and a prime ideal $q \in \operatorname{Min}(A)$ such that $q \subseteq P$. Note that $q \in \operatorname{Spec}(A) \backslash V(I)$. Then we get

$$
\operatorname{depth} A_{q}+\text { height }\left(\frac{I+q}{q}\right)=\operatorname{height}(I+q) \leq \text { height } P=h .
$$

Hence we conclude that $s(I, A) \leq h$, and thus $s(I, A)=h$.

Let $\widehat{A}$ be an m-adic completion of $A$. Then since $\widehat{A}$ is also (F.L.C.), we have $s(I \widehat{A}, \widehat{A})=$ height $I \widehat{A}=h$. Theorem 3.4 implies that $\mathrm{H}_{I}^{h}(A) \otimes \widehat{A} \cong$ $\mathrm{H}_{I \widehat{A}}^{h}(\widehat{A})$ is not of finite type, and neither is $\mathrm{H}_{I}^{h}(A)$.

We now prove Proposition 3.3. We may assume that $I=\sqrt{I}$. (1) (2) follows from Proposition 3.1. Now suppose (1) or (2). We must show that $A / I$ is equidimensional, that is, bight $I=$ height $I$. Now suppose bight $I>$ height $I$. Set

$$
U=\bigcap\left\{P \mid P \in \operatorname{Min}_{A}(A / I), \text { height } P<\operatorname{bight} I\right\}
$$

and

$$
J=\bigcap\left\{P \mid P \in \operatorname{Min}_{A}(A / I) \text {, height } P=\operatorname{bight} I\right\} .
$$

Then we have $I=U \cap J$ and $h+1 \leq$ height $J=$ bight $J \leq d-1$. Localizing at a prime ideal $Q \in \operatorname{Min}_{A}(A / U+J)$, we may assume that $\sqrt{U+J}=\mathfrak{m}$. 
Then from the Mayer-Vietoris sequence of local cohomology, we get as follows :

$$
\mathrm{H}_{\mathrm{m}}^{i}(A) \rightarrow \mathrm{H}_{U}^{i}(A) \oplus \mathrm{H}_{J}^{i}(A) \rightarrow \mathrm{H}_{I}^{i}(A) \quad(\mathrm{ex}) \quad \text { for all } i \leq d-1
$$

By the assumption, we obtain that $\mathrm{H}_{J}^{i}(A)$ is of finite type for all $h+1 \leq$ $i \leq d-1$. By Corollary 3.5, we get a contradiction. Therefore $A / I$ is equidimensional. The proof of the last assertion follows from the similar argument as in Theorem 1.7 and Corollary 2.4 (cf. Hochster and Huneke [9, Theorem 3.3]).

Huneke [10] asked the following question related to Faltings' Theorem.

Question 3.6. ([10, Problem 3.3]) Let $M$ be a finite A-module. Consider the set of integers,

$$
\mathbb{W}=\left\{\operatorname{depth} M_{P}+\text { height }\left(\frac{I+P}{P}\right) \mid P \in \operatorname{Spec}(A) \backslash V(I)\right\} .
$$

Then $n \geq 0$ is not in $\mathbb{W}$ if and only if $\mathrm{H}_{I}^{n}(M)$ is of finite type.

However, we can give the following example.

Example 3.7. Let $A$ be a complete Cohen-Macaulay local domain with $n=\operatorname{dim} A \geq 2$. Let $I$ be an ideal of $A$ such that $\operatorname{dim} A / I=1$. Then $n \in \mathbb{W}$, but $\mathrm{H}_{I}^{n}(A)=0$.

Proof. Take a prime ideal $P \in \operatorname{Spec}(A) \backslash V(I)$ such that $\operatorname{dim} A / P=1$. Then since $\sqrt{I+P}=\mathfrak{m}$, we get

$$
n=\operatorname{dim} A_{P}+\operatorname{dim} A / P \in \mathbb{W} .
$$

On the other hand, we have $\mathrm{H}_{I}^{n}(A)=0$ by 'The Local LichtenbaumHartshorne Theorem.'

Acknowledgement. The author is grateful to the referee for several useful comments. 


\section{REFERENCES}

[1] M. Brodmann and J. Rung, Local cohomology and the connectedness dimension in algebraic varieties, Comment. Math. Helv., 61 (1986), 481-490.

[2] F. W. Call and R. Y. Sharp, A short proof of the Local Lıchtenbaum-Hartshorne Theorem on the vanıshing of local cohomology, Bull. London Math. Soc., 18 (1986), 261-264.

[3] D. Delfino, On the cofiniteness of local cohomology modules, Math. Proc. Camb. Phil. Soc., 115 (1994), 79-84.

[4] G. Faltings, Uber die Annulatoren lokaler Kohomologiegruppen, Arch. Math., 30 (1978), 473-476.

[5] A. Grothendieck, Local cohomology, notes by R. Hartshorne, Lecture notes in Math., 862, Springer-Verlag, 1966.

[6] A. Grothendieck, Cohomologie locale des faisceaux cohérents et théormies de Lefshetz locaux et globaux, S.G.A. II, North-Holland, 1968.

[7] R. Hartshorne, Complete intersections and connectedness, Amer. J. Math., 84 (1962), 497-508.

[8] R. Hartshorne, Affine Duality and Cofiniteness, Invent. Math., 9 (1970), 145-164.

[9] M. Hochster and C. Huneke, Indecomposable Canonıcal Modules and Connectedness, Comp. Math., 159 (1994), 197-208.

[10] C. Huneke, Problems on local cohomology, in Free Resolutions in Commutative Algebra and Algebraic Geometry: Sundance 90 (Edited by D. Eisenbud and C. Huneke).

[11] C. Huneke and J. Koh, Cofiniteness and vanishing of local cohomology modules, Math. Proc. Camb. Phil. Soc., 110 (1991), 421-429.

[12] C. Huneke and G. Lyubeznik, On the vanishing of local cohomology modules, Invent. Math., 102 (1990), 73-93.

[13] K. -i. Kawasaki, On the finiteness of Bass numbers of local cohomology modules, Proc. Amer. Math. Soc. (to appear).

[14] H. Matsumura, Commutative Ring Theory, Cambridge Studies in Adv. in Math., 1986.

[15] M. Nagata, Local Rings, Interscience Tracts in Pure and Appl. Math. 13, Wiley, New York, 1962.

[16] C. Peskine and L. Szpiro, Dimension Projective Finie et Cohomologie Locale, I.H.E.S., 42 (1973), 323-395.

[17] J. Rotman, An Introduction to Homological Algebra, Academic Press, 1979.

Graduate School of Polymathematics

Nagoya University

Chikusa-ku, Nagoya 464-01

Japan

yoshida@math.nagoya-u.ac.jp 